\section{Bertrand Fontaine} Karin Jurkat-Rott

Alexis Elbaz José Valé-Santos Pascale Lapie Emmanuelle Plassart Jocelyne Reboul Claire-Sophie Rime Michel Fardeau Frank Lehmann-Horn

\section{ADRESSES}

B. Fontaine: chef de clinique-assistant. A. Elbaz: interne des hoopilaux de Paris. J. Valé-Santos: interne des hopitaux du Portugal. P. Lapie: chercheur post-doctoral. E. Plassart : étudiante de 3 cycle. J. Reboul: technicienne. C.S. Rime: technicienne. Laboratoire de neurobiologie cellulaire, moléculaire et clinique, Inserm U. 134 et Fédération de neurologie, hôpital Pitié-Salpêtrière, 47-83, boulevard de l'Hôpital, 75651 Paris Cedex 13, France. M. Fardeau: professeur, directeur de l'U. 153 de l'Inserm. Inserm U. 153, hôpital Pitié-Salpêtrière, 47-83, boulevard de l'Hôpital, 75651 Paris Cedex 13, France. K. Jurkat-Rout: étudiante de 3 grle. F. Lehmann-Horn: professeur de physiologie. Abteilung für Angewandte Physiologie, Ulm Universität, 89081 Ulm, Allemagne.

TIRÉS À PART

B. Fontaine.

\section{Paralysie périodique hypokaliémique et récepteur des dihydropyridines: un rôle inattendu pour un canal calcium}

Les paralysies périodiques et les myotonies sont des maladies des canaux ioniques musculaires. La paralysie périodique hypokaliémique (hypoPP), caractérisée par des accès réversibles de paralysie musculaire contemporains d'une chute de la kaliémie, était la dernière affection de ce groupe de maladies dont le gène restait inconnu. Par une stratégie associant un criblage anonyme du génome et une approche par "gènes candidats ", le locus hypoPP et le gène codant pour un canal calcium, la sous-unité $\alpha 1$ du récepteur des dihydropyridines, ont été génétiquement confondus et localisés sur le bras long du chromosome 1 en 1q31-32. Deux mutations, rendant compte de la majorité des cas d'hypoPP, ont été retrouvées dans les segments $\mathrm{S} 4$ des domaines II et IV du récepteur des dihydropyridines, dont le rôle est d'assurer la sensibilité du canal calcium aux différences de potentiel. Elles pourraient donc expliquer les accès de paralysie. En revanche, leur responsabilité directe dans la survenue de l'hypokaliémie et de la myopathie vacuolaire reste encore mal comprise.

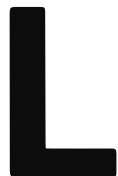

es paralysies périodiques et les myotonies, à l'exception de la myotonie dystrophique de Steinert, sont des maladies héréditaires des canaux ioniques musculaires. Décrites depuis une centaine d'années, elles n'ont fait l'objet de classification claire que depuis les années 1990, grâce à l'identification des gènes incriminés. Elles représentent un des succès de l'approche par "gènes candidats" [1]. Elles illustrent les deux révolutions que connaît actuellement la re- cherche neurologique moléculaire: (1) l'identification de gènes grâce à une connaissance accrue du génome et (2) le bouleversement de la nosologie neurologique par l'introduction des gènes anormaux au sein des classifications anatomo-cliniques.

Contraction musculaire et maladies des canaux ioniques

La contraction musculaire nécessite l'intégrité de différents mécanismes: la transmission du message du moto- 


\section{RÉFÉRENCES}

1. Fontaine B. Étude génétique et moléculaire des paralysies dyskaliémiques familiales un succès de l'approche par gènes candidats. médecine/sciences $1991 ; 8$ : 41-5.

2. Fontaine B. Periodic paralysis, myotonia congenita and sarcolemmal ion channels: a success of the candidate gene approach. Neuromuse Dis 1993; 3: 101-7.

3. Rüdel R, Ricker K, Lehman-Horn F. Genotype-phenotype correlations in human skeletal muscle sodium channel diseases. Arch Neurol 1993; 50 : 1241-8.

4. Ptacek LJ, Johnson KJ, Griggs RC. Genetics and physiology of the myotonic muscle disorders. N Engly Med 1993; 328 : 482-9.

5. Hoffman EP, Wang J. Duchenne-Becker muscular dystrophy and the nondystrophic myotonias. Arch Neurol 1993; 50 : 1227-37.

6. McClatchey AI, McKenna-Yasek D, Cros D, Worthen HG, Kuncl RW, DeSilva SM, Cornblath DR, Gusella JF, Brown Jr RH. Novel mutations in families with unusual and variable disorders of the skeletal muscle sodium channel. Nature Genet 1992; 2: 148-52.

7. Barchi RL. Sodium channel gene defects in the periodic paralysis. Curr $(2 p$ Neurobiol $1992 ; 2: 631-7$.

8. Plassart E, Reboul J, Rime CS, Recan D, Millasseau $\mathrm{P}$, et al. Mutations in the muscle sodium channel gene (SCN4A) in 13 French families with hyperkalemic periodic paralysis and paramyotonia congenita: phenotype to genotype correlations and demonstration of the predominance of two mutations. Eur J Hum Genet 1994; 2 : 110-24.

9. Heine R, Pika U, Lehmann-Horn F. A novel SCN4A mutation causing myotonia aggravated by cold and potassium. Hum Mol gravated by cold and pot
Genet $1993 ; 2: 1349-13$.

10. Ptacek LJ, Tawil R, Griggs RC, Meola G, McManis $\mathrm{P}$, et al. Sodium channel mutations in acetazolamide-responsive myotonia congenita, paramyotonia congenita and hyperkalemic periodic paralysis. Neurology $1994 ; 44: 1500-3$

11. Abdalla JA, Casley WL, Cousin KH, Hudson A], Murphy EG, Cornélis FC, Hashimoto L, Ebers GC. Linkage of Thomsen disease to the T-cell-receptor beta (TCRB) locus on chromosome $7 \mathrm{q} 35$. Am / Hum Genet $1992 ; 51: 579-84$.

12. Koch MC, Steinmeyer K, Lorenz C, Ricker K, Wolf F, Otto M, Zoll B, LehmannHorn F, Grzeschik KH, Jentsch TJ. The skeletal muscle chloride channel in dominant and recessive human myotonia. Science 1992 ; 257 : 797-800

13. George AL Jr, Crackower MA, Abdalla JA, Hudson AJ, Ebers GC. Molecular basis of Thomsen's disease (autosomal dominant myotonia congenita). Nature Genet 1993; 3 : 305-9.

14. Nargeot J, Charnet P. Diversité moléculaire des canaux calciques: du gène à la fonction. médecine/sciences 1994; $10: 1293-308$.

15. Cannon SC, Brown RH, Corey DP. A sodium channel defect in hyperkalemic periodic paralysis: potassium-induced failure of

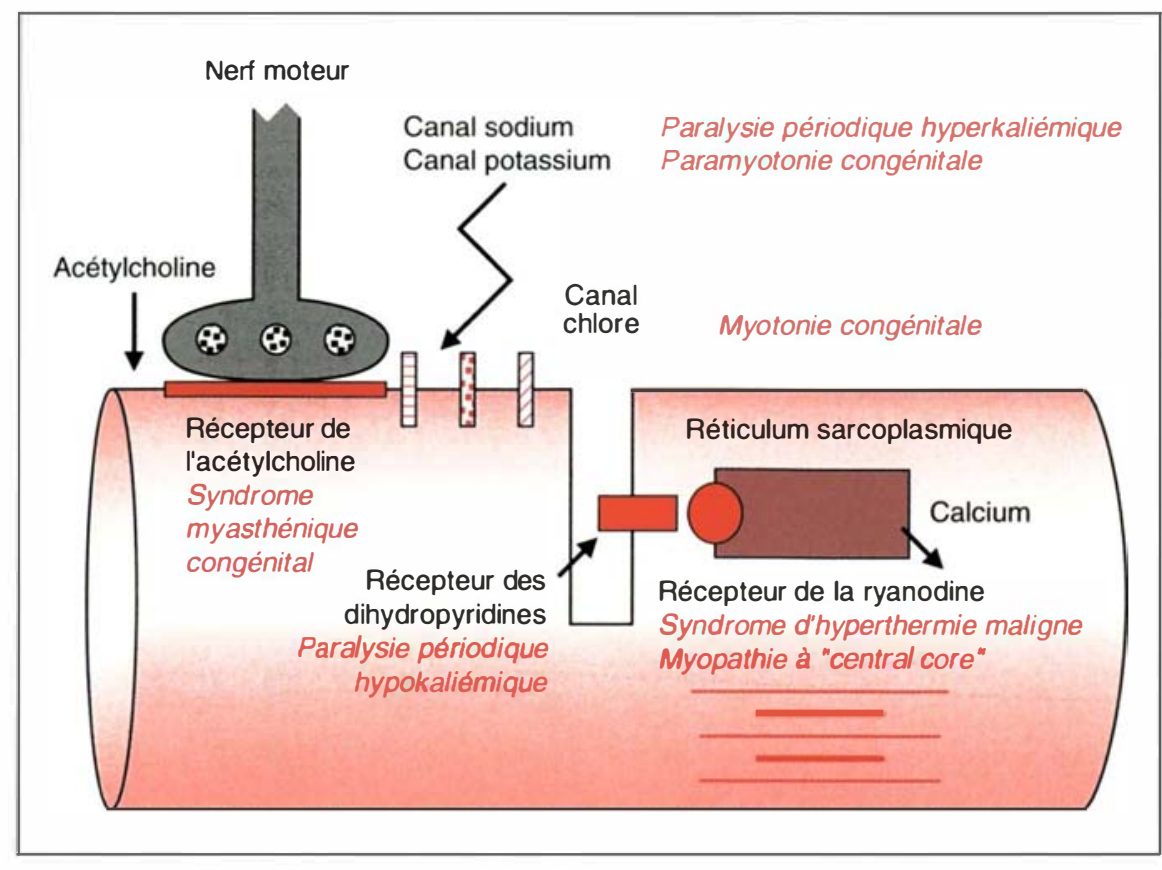

Figure 1. Contraction du muscle squelettique et maladies musculaires. Les myasthénies congénitales, caractérisées par un épuisement progressif et rapide de la force musculaire des muscles volontaires, sont dues à des altérations de la jonction neuro-musculaire (récepteur de l'acétylcholine); la paralysie périodique hyperkaliémique et la paramyotonie congénitale sont toutes deux dues à des mutations du canal sodium des muscles squelettiques $(\mathrm{m} / \mathrm{s}$ $\mathrm{n}^{\circ} 1$, vol. 7, p. 79) ; la myotonie congénitale est due à une anomalie moléculaire du canal chlorure (m/s $\mathrm{n}^{\circ}$ 6-7, vol. 9, p. 805); le syndrome d'hyperthermie maligne et la myopathie à central core sont tous deux la conséquence de mutations du récepteur de la ryanodine $\left(\mathrm{m} / \mathrm{s} \mathrm{n}^{\circ} 11\right.$, vol. $\left.9, \mathrm{p} .1278\right)$; la paralysie périodique hypokaliémique provient de mutations du récepteur des dihydropyridines qui est aussi un canal calcium.

neurone au muscle grâce à une synapse chimique dont le neurotransmetteur est l'acétylcholine, la propagation du potentiel d'action au niveau de la membrane musculaire grâce à l'ouverture et à la fermeture séquentielles des canaux sodium et potassium, enfin le couplage excitation-contraction au niveau du système tubulaire et du réticulum sarcoplasmique (figure 1). L'altération de l'un de ces mécanismes peut être responsable de maladie musculaire comme l'illustre la figure 1. Les paralysies périodiques se manifestent par des accès réversibles de paralysie musculaire dus à une dépolarisation anormalement prolongée de la membrane musculaire. Selon les variations du taux sanguin de potassium au cours des crises, on a pu distinguer deux types de paralysie périodique : hyperkaliémique et hypokalié- mique. Ia myotonie se traduit par une difficulté à la décontraction musculaire en rapport avec une hyperexcitabilité de la membrane musculaire. I.a myotonie congénitale est améliorée par l'effort ("phénomène d'échauffement») au contraire de la paramyotonie congénitale ou "myotonie paradoxale" qui est, quant à elle, aggravée par l'effort musculaire. La sensibilité au froid plus importante de la paramyotonie congénitale distingue également cette dernière de la myotonie congénitale. Paramyotonie congénitale et paralysie périodique hyperkaliémique sont dues à des mutations alléliques du canal sodium SCN4A (m/s $n^{\circ} 5$, vol. $\left.8, p .505\right)$ [1-10]. Les myotonies congénitales sont des maladies du canal chlore CCL.1 (m/s $n^{\circ} 8$, vol. 8, p. 872) [1113]. Les maladies du canal sodium sont de mode de transmission auto- 
somique dominant. Les maladies du canal chlore ont un mode de transmission autosomique récessif ou dominant. La physiopathologie de ces affections est maintenant mieux comprise. Le canal sodium, comme les autres membres de la famille des " canaux ioniques dépendants du voltage ", peut être activé puis inactivé lors des variations de potentiel de la membrane musculaire [14]. Le potentiel d'action active les canaux sodium en dépolarisant la membrane musculaire. Le pore ionique s'ouvre et la conductance sodique s'accroît. L'activation du canal sodium entraîne, cette fois-ci de façon indépendante des variations du potentiel de la membrane musculaire, l'inactivation du canal, c'est-à-dire la fermeture du pore ionique et la diminution de la conductance sodique. Les mutations de SCN4A mises en évidence dans les maladies du canal sodium agiraient surtout en modifiant les paramètres d'inactivation du canal sodium, donc sa fermeture. En cas de défaut de fermeture peu important, une hyperexcitabilité de la membrane musculaire apparaît "par fuite d'ions sodium" se traduisant cliniquement par une myotonie. Si le défaut de fermeture est plus important, la différence de potentiel nécessaire à l'ouverture des canaux sodium peut être atteinte, entrainant une dépolarisation de la membrane musculaire, responsable d'un accès de paralysie [15-21]. La périodicité des accès, le rôle du froid et de l'hyperkaliémie ne sont encore qu'incomplètement compris mais pourraient être en rapport avec une modulation des propriétés électrophysiologiques du canal par son environnement extracellulaire, soit directement, ce qui n'a pas pu être démontré lors des expériences d'expression in vilro des mutations, soit plus vraisemblablement indirectement, par l'intermédiaire d'une interaction avec d'autres canaux ioniques par exemple. Il faut rappeler qu'au cours de l'effort musculaire, l'équilibre acido-basique est modifié et la concentration extracellulaire de potassium croît en raison du métabolisme énergétique du muscle, modifiant ainsi l'environnement extracellulaire des canaux ioniques. Le rôle du canal chlore est de maintenir le potentiel de repos de la membrane musculaire en permettant les flux passifs d'ions chlore induits par les différences des concentrations extracellulaire et intracellulaire des ions sodium et potassium, et leur modification lors de la conduction du potentiel d'action. On conçoit donc qu'une absence complète de canal chlorure fonctionnel, comme dans la forme récessive de la maladie, entraîne une hyperexcitabilité de la membrane musculaire se traduisant cliniquement par une myotonie [22] Dans la forme dominante de la maladie, il y a production à la fois de canaux chlore normaux et mutés. Le canal chlore n'est fonctionnel que sous la forme d'un complexe oligomérique formé par quatre canaux chlore $\left(m / s \quad n^{\circ} 5\right.$, vol. $10, p$. 603) Lescanaux mutés inactivent le complexe oligomérique même si celui-ci contient des canaux normaux [23, 24].

Parmi les maladies musculaires des canaux ioniques, la paralysie périodique hypokaliémique (hypoPP) était le dernier grand syndrome orphelin de gène. L'hypoPP se manifeste par des accès de paralysie musculaire durant de quelques heures à 48 heures. Ils touchent le plus souvent les quatre membres, provoquant alors une tétraplégie. Ils peuvent n'être que partiels, n'impliquant qu'un membre ou qu'un segment de membre, ou plus étendus, intéressant alors la musculature à innervation bulbaire et les muscles respiratoires, habituellement respectés. La maladie débute en général au cours de la seconde décemnie. Le mode de transmission est autosomique dominant. La pénétrance est incomplète, particulièrement cher la femme. Des cas sporadiques ainsi que des néo-mutations ont été rapportés. La gravité de la maladie provient, soit de l'importance de la chute de la kaliémie qu peut induire un trouble du rythme cardiaque, soit de la myopathie vacuolaire qui se développe au cours de la quatrième ou de la cinquième décennie. La gravité de la myopathie vacuolaire est variable et sa survenue est indépendante de la fréquence ou du nombre des accès de paralysie périodique. La myopathie vacuolaire peut même survenir chez un sujet n'ayant jamais présenté d'accès de paralysie périodique. Elle est responsable d'un déficit moteur qui, cette fois-ci, est permanent. Au contraire de la paralysie périodique hyperkaliémique, on n'observe jamais de manifestation myotonique dans l'hypoPP. Comme pour la paralysie périodique hyperkaliémique, les accès de paralysie périodique s'accompagnent d'une dépolarisation de la membrane musculaire. Cette dernière a été considérée comme "paradoxale" car elle est contemporaine d'une hypokaliémie dont l'effet habituel est d'hyperpolariser la cellule musculaire. Ainsi, si l'on maintient vivantes des fibres musculaires de patient in vitro, on observe l'apparition d'une dépolarisation musculaire en diminuant la concentration de potassium extracellulaire [25]. Ce comportement étonnant des fibres musculaires de patients hypoPP ne permettait pas de proposer un gène candidat comme dans le cas des maladies du canal sodium. Néanmoins, il incitait à chercher le gène défectueux parmi les canaux ioniques et à aborder l'étude des hypoPP par une stratégie "gènes candidats". C'est ce qui fut réalisé de façon infructueuse pour les canaux sodium et chlore [26-28]. Les canaux potassium furent également testés sans succès. C'est pourquoi, toujours persuadés du rôle possible d'un canal ionique, nous décidâmes d'élargir notre recherche à d'autres canaux ioniques.

\section{La famille des crcanaux ioniques dépendants du voltage "}

Elle comprend les canaux potassium, sodium et calcium. Ces canaux ioniques partagent une structure commune faite de six segments transmembranaires composant un domaine [14] (figure 2). Ces canaux ont été fortement conservés au cours de l'évolution, probablement en raison de leur rôle dans les processus de communication intercellulaire. Par exemple, la conservation des acides aminés est plus importante entre mêmes canaux ioniques appartenant à des espèces différentes, qu'entre les différents canaux ioniques d'une même famille au sein d'une même espèce. Cette conservation remarquable remonte à au moins 800 millions d'années puisqu'elle concerne des espèces aussi différentes que la drosophile, le poisson torpille ou les mammifères (souris, lapin, rat, homme). Elle serait 


\section{RÉFÉRENCES}

16. Lehmann-Horn F, Iazzo PA, Hatt $H$ Franke C. Altered gating and conductance of $\mathrm{Na}^{+}$channels in hyperkalemic periodic paralysis. Plugers Arch 1991 ; 418: 297-9.

17. Cannon SC, Brown RH, Corey DP. Theoretical reconstruction of myotonia and paralysis caused by incomplete inactivation of sodium channels. Biophys / 1993; 65: 270-88.

18. Cannon SC, Strittmatter SM. Functional expression of sodium channel mutations identified in families with periodic paralysis. Neuron $1993 ; 10$ : 317-26.

19. Cummins TR, Zhou J, Sigworth FJ, Ukomadu C, Stephan M, Ptacek LJ, Agnew WS. Functional consequences of a $\mathrm{Na}^{+}$channel mutation causing hyperkalemic periodic paralysis. Neuron 1993;10: 667-78.

20. Chahine M, George AL Jr, Zhou M, Ji S, Sun W, Barchi RI, Horn R. Sodium channel mutations in paramyotonia congenita uncouple inactivation from activation. Neuron 1994 ; 12: $281-94$.

21. Lerche H, Heine R, Pika U, George AI, Mitrovic N, el al. (12 auteurs). Human sodium channel myotonia: slowed channel inactivation due to substitutions for a glycine within the III/IV linker. I Physiol 1993; 470 : within

22. Fahlke C, Zachar E, Rüdel R. Chloride channels with reduced single-channel conductance in recessive myotonia congenita. Neuron $1993 ; 10: 225-32$

23. Steinmeyer K, Lorenz C, Pusch M, Koch MC, Jentsch TJ. Multimeric structure of CLC. - chloride channel revealed by mutations in dominant myotonia congenita (Thomsen). EMBO J 1994; 13: 737-43.

24. Heine R, George AL Jr, Pika U, Deymeer F, Rüdel R, Lehmann-Horn F. Proof of a non-functional muscle chloride channel in recessive myotonia congenita (Becker) by detection of a 4 base pair deletion. Hum Mol Genet 1994; 3 ; 1123-8.

25. Rüdel R, Lehmann-Horn F, Ricker K Küther G. Hypokalemic periodic paralysis in vitro investigations of muscle fiber parameters. Muscle Nerve 1984; 7: 110-20.

26. Fontaine B, Khurana TS, Hoffman EP, Bruns GAP, Haines JL, et al. (15 auteurs). Hyperkalemic periodic paralysis and the adult muscle sodium channel $\alpha$-subunit gene. Science 1990; 250 : 1000-2.

27. Fontaine B, Troffater J, Rouleau GA Khurana TS, Haines J, Brown R, Gusella J. Different gene loci for hyperkalemic and hypokalemic periodic paralysis. Neuromusc Dis 1991 ; 1 : 235-8.

28. Casley WL, Allon M, Cousin HK, Ting SS, Crackower MA, Hashimoto L, Cornélis F, Beckmann IS, Hudson AJ, Ebers GC. Ex clusion of linkage between hypokalemic periodic paralysis (Hokpp) and three candidate loci. Genomics 1992; 14: 493-4.

29. Milkman R. An Escherichia coli homologue of eukaryotic potassium channel proteins même antérieure à la divergence entre eucaryotes et procaryotes survenue il y a environ 1,4 milliard d'années car un canal potassium de structure identique à celui des eucaryotes a été récemment isolé chez une bactérie [29]. Un canal ionique fonctionnel est réalisé par le regroupement de quatre domaines qui permettent la constitution d'un pore ionique. Les gènes des canaux potassium ne codent que pour un seul domaine, à la différence des gènes des canaux sodium et calcium qui codent pour les quatre domaines. Il faut donc le produit de quatre gènes pour former un canal potassium fonctionnel et d'un gène seulement pour les canaux sodium et calcium. L'ensemble de ces gènes a probablement évolué à partir d'un ancêtre commun proche des gènes codant pour les canaux potassiques tels qu'ils peuvent être observés aujourd'hui. Il faut alors postuler que des événements de duplication génique sont intervenus pour souder les quatre domaines en un même gène pour les canaux sodique et calcique. Cette hypothèse de duplication génique est confortée par l'observation de l'organisation chromosomique des canaux ioniques. En effet, un regroupement de gènes codant pour des canaux sodium a été mis en évidence chez la souris et chez l'homme sur le chromosome 2 .

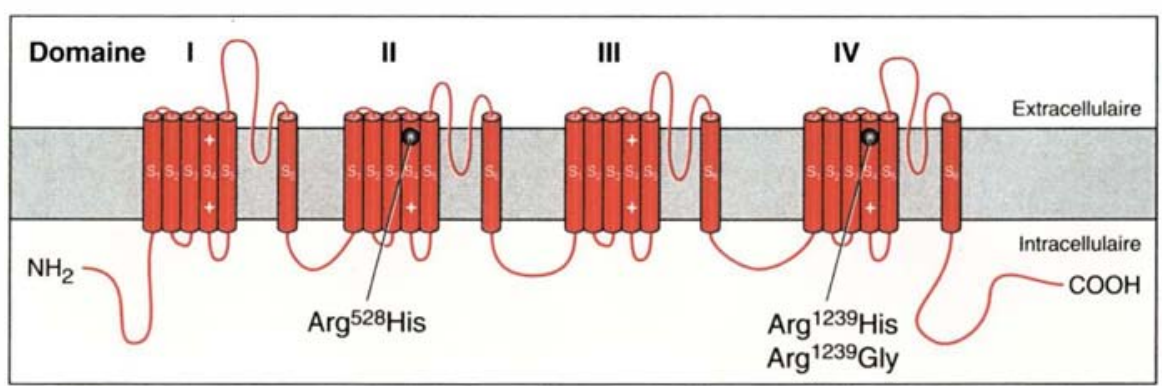

Figure 2. Sous-unité $\alpha 1$ du récepteur des dihydropyridines llocus CACNL1A3) et mutations responsables de paralysie périodique hypokaliémique (hypoPP). Les canaux ioniques partagent une structure commune faite de six segments transmembranaires composant un domaine [14]. L'étude de protéines mutées ou de canaux chimères réalisée à partir des différentes sous-unités $\alpha 1$ clonées a permis de localiser certaines séquences importantes pour le fonctionnement normal du canal. Le segment sensible au voltage, responsable de l'activation, a ainsi pu être identifié comme le quatrième segment de chaque domaine (S4). Les substitutions d'acides aminés retrouvées dans les hypoPP mettent en jeu des résidus très conservés, aussi bien dans les canaux sodium que dans les canaux calcium. Ces mutations modifieraient les conditions d'inactivation du récepteur (Arg ${ }^{528} \rightarrow$ His), ou conduiraient à une diminution de la conductance du canal (Arg ${ }^{1239_{-}}$. His) [42].
Forts de l'ensemble de ces données, nous avons décidé de sélectionner
sur le génome humain les régions chromosomiques contenant un canal ionique, ce qui représentait de $18 \%$
à $20 \%$ du génome. Le pari était le suivant: si nous localisons le gène gène hypoPP aura toutes les chances d'être, soit le canal ionique ainsi mis inattendu pour un canal ionique déjà conn soit un autre canal ionique tageant peut-être avec ce dernier une filiation par duplication génique. est la premi

\section{Le gène des hypopP est un canal calcium, le récepteur CACNL1A3, localisé sur le bras long du chromosome 1}

L'étude de liaison génétique initiale différente: portugaise, allemande fouçaise. Nous observâmes des lodtués sur le bras long du chromosome 1 (figure 3). Les lod-scores étaient par exemple de 8,98 pour une frac- 


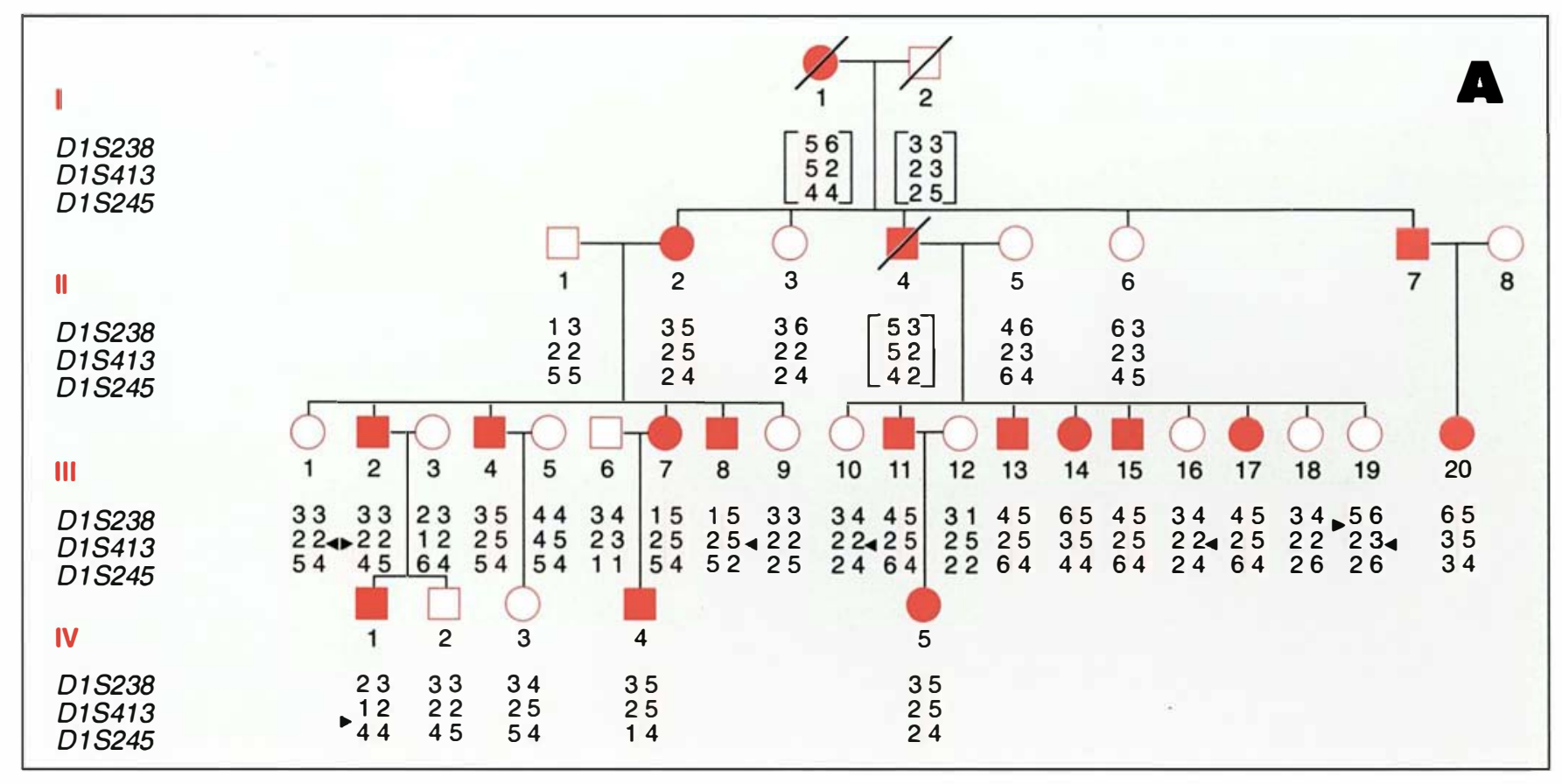

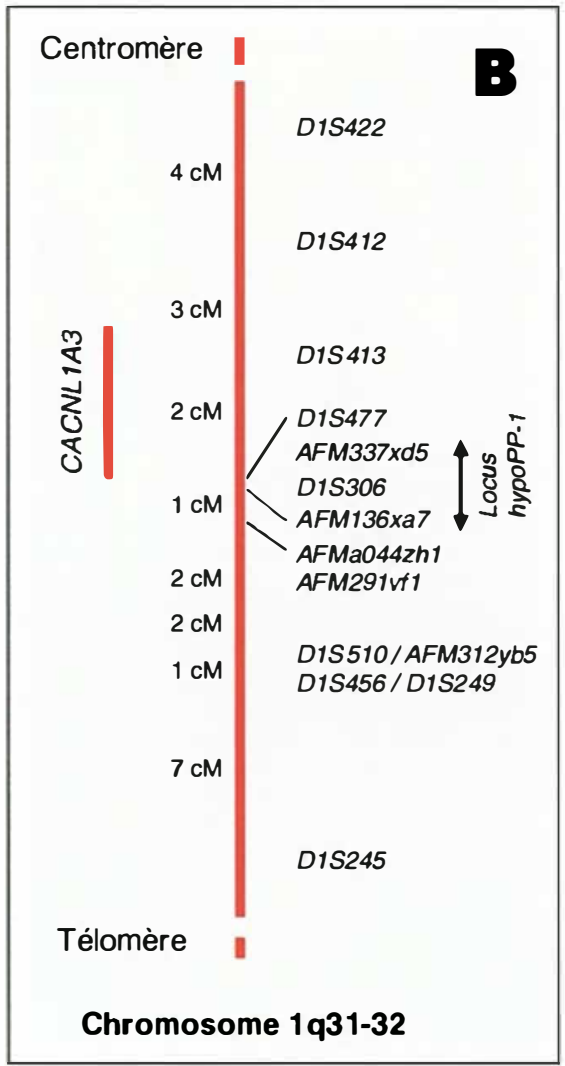

$m / s n^{\circ} 7$, vol. 11, juillet 95

Figure 3. Étude de liaison génétique dans la paralysie périodique hypokaliémique (hypoPP). A. Famille d'origine portugaise ayant permis d'établir la liaison génétique entre le gène hypoPP et des marqueurs du chromosome 1. Le nom des différents marqueurs utilisés est donné dans la colonne de gauche. L'haplotype ségrégeant avec la maladie est ombré. Les pointes de flèche indiquent les zones de recombinaison. En rouge, les sujets atteints; les symboles barrés correspondent aux sujets décédés. B. Carte génétique de la région du chromosome 1q31-32. La position des loci CACNL1A3 et hypoPP-1 est indiquée. Les marqueurs bordants le locus hypoPP-1 et leurs distances génétiques sont également indiqués.

tion de recombinaison $\theta=0,02$ pour le marqueur ID1S413 [30]. Ils étaient franchement supérieurs au seuil de 3,00 habituellement utilisé pour affirmer une liaison génétique. L’analyse "multipoint" et celle des recombinants permit de préciser que le gène hypoPP était localisé dans un intervalle de $8 \mathrm{cM}$ défini par les marqueurs I)1S413 et D1S249 (figure 3) [30]. Dans cette même région du génome avait été localisé le gène codant pour la sous-unité $\alpha$ l du récepteur des dihydropyridines (locus CACNL1A3) $[31,32]$. A l'aide d'un microsatellite intragénique au récepteur des dihydropyridines, nous vérifiâmes qu'il existait bien un intervalle de $5 \mathrm{cM}$ commun aux loci hypoPP et CACNL1A3 (figure 3) [30]. Nous vérifiâmes, en outre, la coségrégation d'un allèle du récepteur des dihydropyridines avec la maladie [30]. Le lod-score était de 5,24 pour une fraction de recombinaison $\theta=0,00[30]$. L'analyse de liaison génétique indi- qua donc que les loci hypoPP et CACNLIA3 étaient colocalisés sur le chromosome 1 en 1q31-32 (figure 3) et que le récepteur des dihydropyridines était un gène candidat pour l'hypoPP [30]. Cette localisation génétique a été par la suite affinée par la description de marqueurs permettant de mieux définir la position du locus hypoPP $[33,34]$.

L'étape suivante consistait à chercher une mutation dans la séquence codante du récepteur des dihydropyridines. Après transcription inverse de l'ARN, des ADNc musculaires furent préparés à partir de biopsies musculaires de patients atteints d'hypoPP et de témoins. Les fragments d'ADNc furent obtenus par réaction de polymérisation en chaîne (PCR) en utilisant des amorces spécifiques de la séquence humaine. La séquence nucléotidique de ces fragments fut réalisée et il fut ainsi mis en évidence des modifications nucléotidiques par rapport à la 


\section{RÉFÉRENCES}

30. Fontaine B, Vale-Santos I, Jurkat-Rott K, Reboul J, Plassart E, et al. (13 auteurs). Mapping of hypokalemic periodic paralysis (hy-
poPp) to chromosome lq31-32 in three EupoPP) to chromosome lq $31-32$ in three Eu-
ropean families. Nature Genet $1994 ; 3$ : 267-72.

31. Gregg RG, Cough F, Hogan K, Powers PA. Assignment of the human gene for the $\alpha 1$ subunit of the skeletal muscle DHP-sensitive Subunit of the skeletal muscle DHP-sensitive
$\mathrm{Ca}^{2+}$ channel (CACNL.1A3) to chromosome 1q31-32. Genomics 1993; 15: 107-12.

32. Drouet B, Garcia L, Simon-Chazottes D, Chazottes D, Mattei MG, Guenet JL, Schwartz A, Varadi G, Pinçon-Raymond M. The gene coding for the alphal subunit of the skeletal dihydropyridine receptor maps to mouse chromosome 1 and human lq32. Mamm Genome 1993; 4 : 499-503

33. Plassart E, Elbaz A, Vale-Santos J, Reboul I, Lapie P, et al. (12 auteurs). Genetic heterogeneity in hypokalemic periodic paralysis (hypoPP). Hum Genet 1994; 94: 551-6.

34. Jurkat-Rott K, Lehmann-Horn F, Elbaz A, Heine R, Gregg RG, et al. (11 auteurs). A calcium channel mutation causing hypokalemic periodic paralysis. Hum Mol Genet $1994 ; 3: 1415-9$.

35. Ptacek LJ, Tawill R, Griggs RC, Engel AG, Layzer RB, et al. (12 auteurs). Dihydropyridine receptor mutations cause hypokalemic periodic paralysis. Cell 1994; 77: 863-8.

36. Tanabe TH, Takeshima H, Mikami V, et al. Primary structure of the receptor for calcium channel blockers from skeletal cium channel blockers from
muscle. Nature 1987;328:313-8.

37. Catterall WA. Structure and function of voltage-gated ion channels. Trends Neurosit $1993 ; 16: 500-6$

38. Lory P, Varadi G, Schwartz A. Molecular insights into regulation of L-type calcium channel function. Trends Neurosi 1991; 6: channel

39. Tanabe T, Beam KG, Powell JA, Numa $S$. Restoration of excitation-contraction coltpling and slow calcium current in dysgenic muscle by dihydropyridine receptor complementary DNA. Nature $1988 ; 336: 1349$.

40. Pinçon-Raymond $M$, Vicart $P$, Bois $P$, Chassande $O$, Romey $G$, Varadi $G$, Li ZL, Ladzunski M, Rieger F, Paulin D. Conditional immortalization of normal and dysgenic mouse muscle cells by the SV40 large T antigen under the vimentin promoter control. Dev Biol 1991 ; 148 : $517-28$.

41. Chaudhari N. A single nucleotide deletion in the skeletal muscle-specific calcium channel transcript of muscular dysgenesis (mdg) mice. I Biol Chem 1992; 267 : 25636-9.

42. Sipos I, Yurkat-Rott K, Harasztosi CS, Fontaine B, Kovacs L, Melzer W, LehmannHorn F. Skeletal muscle DHP receptor mutations alter calcium currents in human hypokalemic periodic paralysis myotubes. $J$ Physiol $1995 ; 483: 299-306$

43. Elbar A, Vale-Santos J, Jurkat-Rott K, et al. Hypokalemic periodic paralysis and the dihydropyridine receptor: genotype/phenotype correlations for two predominant mutations and evidence for the absence of a founder effect in 16 Caucasian families. Am J Hum Ge séquence normale. Environ $80 \%$ de l'ADNc, dont les quatre domaines transmembranaires, furent étudiés. Certains changements nucléotidiques étaient conservateurs en terme d'acide aminé, ou retrouvés chez les témoins. Ils furent donc considérés comme des polymorphismes de l'ADN sans rapport avec la maladie. En revanche, la transition d'un $G$ en A entraînait le changement d'une arginine en histidine en position 528 [34]. Cette position est située dans le quatrième segment du second domaine (figure 2). Elle est conservée aussi bien dans les canaux sodium que dans les canaux calcium. Le changement nucléotidique modifie une charge positive dans le quatrième segment dont le rôle est de donner au canal une sensibilité aux différences de potentiel de la membrane musculaire [14]. Ce changement nucléotidique était un bon candidat pour représenter une mutation. Nous avons alors déterminé un couple d'amorces permettant une étude de l'ADN génomique et mis en évidence un site de restriction modifié par la mutation, ce qui permettait une étude rapide des familles [34]. Nous pûmes ainsi montrer que le changement nucléotidique conduisant au changement d'acide aminé $\operatorname{Arg}^{528} \rightarrow$ His remplissait toutes les conditions d'une mutation car: (1) il n'était observé que chez les patients et pas chez les sujets sains ; (2) il coségrégeait avec la maladie au sein des familles ; (3) il était mis en évidence dans un nombre important de familles d'hypoPP (40\%) et (4) il était responsable d'un changement non conservateur d'un acide aminé dans une région conservée et fonctionnellement importante de la protéine [34]. Le dernier critère aurait été la démonstration d'une néomutation. Ce dernier n'a pu être mis en évidence au sein des familles que nous avons étudiées [34]. Dans le même temps, une équipe américaine rapportait une mutation en position 1239 dans le segment 4 du domaine IV responsable, de façon similaire à la nôtre, du remplacement d'une arginine en histidine dans la majorité des familles, ou en glycine, plus rarement (figure 2) [35]. La mutation Arg ${ }^{12339} \rightarrow$ His a été mise en évidence dans $30 \%$ des familles hypoPP analysées par l'équipe américaine [35].
Nous avons donc démontré le rôle causal inattendu d'un canal calcium dans l'hypoPP: le récepteur des dihydropyridines. Ce récepteur des dihydropyridines est un complexe protéique composé de plusieurs sous-unités: $\alpha$ l (locus CACNL1A3), $\alpha 2, \beta, \gamma$ et $\delta[36,37]$. La plupart des propriétés du récepteur des dihydropyridines peuvent être reconstituées in vitro par l'expression de la sousunité $\alpha \mathrm{l}$, responsable notamment des propriétés d'activation et d'inactivation du canal par le voltage et de fixation des bloquants des canaux calcium comme les dihydropyridines [14]. Le rôle de la sous-unité $\beta 1$ serait de moduler les propriétés d'inactivation du canal calcium [38]. Au plan électrophysiologique, le récepteur des dihydropyridines intervient dans la genèse des courants calciques de type L [14]. Il a un rôle de canal calcium et de couplage entre l'excitation et la contraction musculaire. Pour cette dernière fonction, il interagit probablement avec le récepteur de la ryanodine, autre gène impliqué dans les maladies neuromusculaires $\left(\mathrm{m} / \mathrm{s} n^{\circ} 11\right.$, vol. 9, p. 1278) (figure 1). L'expression de la sous-unité $\alpha$ l du récepteur des dihydropyridines est abolie chez le mutant $m d g$ de la souris $[39,40]$. Le phénotype est celui d'une mutation létale de mode de transmission autosomique récessif entraînant une absence de couplage excitationcontraction. La mutation en cause est une mutation non sens, aboutissant à une terminaison précoce de la traduction de l'ARN messager [41]. Quels peuvent être les mécanismes d'action des mutations mises en évidence dans l'hypoPP? Des résultats préliminaires obtenus sur des fibres musculaires de patients hypoPP maintenues vivantes in vitro indiquent que la mutation $\mathrm{Arg}^{528} \rightarrow$ His modifierait les propriétés d'inactivation de CACNL1A3 [42]. La mutation $\operatorname{Arg}^{1239} \rightarrow$ His pourrait avoir un mécanisme d'action plus complexe, conduisant à une diminition de la conductance du canal [42]. Il est clair qu'un défaut d'inactivation du canal calcium peut entraîner une paralysie musculaire en maintenant une dépolarisation musculaire anormalement prolongée et/ou en interagissant avec le couplage-excitation. A ce stade des recherches, il est diffi- 
cile d'expliquer l'hypokaliémie survenant lors des accès de paralysic musculaire. On peut proposer l'hypothèse suivante: le récepteur des dihydropyridines est localisé dans une région particulière du muscle, le système tubulaire, où il interagit peut-être avec un canal potassium dont l'ouverture entraînerait l'hypokaliémie. Ces mécanismes restent spéculatifs et devront être testés dans un modèle in vitro de la maladie qui est en cours de réalisation.

Après avoir montré que le récepteur des dihydropyridines est responsable d'hypoPP, il reste à répondre à un certain nombre de questions. La première d'entre elles est de savoir si le récepteur des dihydropyridines est le seul gène impliqué dans l'hypoPP. A ce jour, nous avons étudié dix-huit familles d'hypoPP. Deux familles, l'une d'origine française et l'autre d'origine portugaise, ne sont pas liées à hypoPP-1/CACNL,1A3 [33]. Il existe donc un second locus hypoPP même si le locus hypoPP-1/ C.ACNL,1A3 semble rendre compte de la majorité des familles étudiées. Les dix-sept autres familles sont liées au locus hypoPP-1/CACNLIA3. L'une d'entre elles ne possède aucune des mutations actuellement décrites dans le récepteur des dihydropyridines. Il reste donc à découvrir de nouvelles mutations du récepteur des dihydropyridines. Les deux mutations $\operatorname{Arg}^{528} \rightarrow$ His et $\operatorname{Arg}^{1239} \rightarrow$ His - semblent donc rendre compte de la majorité des cas d'hypoPP, permettant de proposer un diagnostic moléculaire facilité par une technique rapide utilisant l'amplification d'AIDN génomique et la coupure par une enzyme de restriction [43].

\section{Conclusion et perspectives}

Par une stratégie associant un criblage anonyme du génome et une approche par "gènes candidats", nous avons pu montrer qu'un canal calcium, la sous-unité $\alpha 1$ du récepteur des dihydropyridines, est le gène responsable des hypoPP. Cette découverte est inattendue car aucune étude électrophysiologique n'avait mis en avant le rôle éventuel de ce gène dans les hypoPP. La connaissance du génome humain progressant rapidement, notre étude incite à privilégier ce type d'approche avant d'envisager une stratégie de type "clonage positionnel" pour l'identification d'un gène responsable de maladie neuromusculaire. De nombreuses questions concernant la physiopathologie de l'hypoPP restent non résolues. Autant l'implication d'un canal calcique permet de rendre compte des accès de paralysie périodique, autant il est difficile d'expliquer l'hypokaliémie. L'expression in vitro des mutations du récepteur des dihydropyridines devrait permettre de répondre à cette question et de tester des agents pharmacologiques modifiant la fonction des canaux calcium dans un but thérapeutique. Par ailleurs, la compréhension de l'implication d'un canal calcium dans la physiopathologie de la myopathie présentée par les patients hypoPP pourrait également avoir des retombées sur la stratégie du traitement des myopathies. D'un point de vue plus général, l'étude de l'expression in vitro de ces mutations naturelles devrait accroître notre connaissance du fonctionnement des canaux calcium et, peut-être, ouvrir de nouvelles perspectives pour les maladies impliquant un fonctionnement anormal d'un canal calcium

\section{Remerciements}

Nous remercions les familles pour leur soutien et les médecins qui nous ont adressé les patients car ce travail n'aurait pas été possible sans leur contribution. Plus particulièrement: les Drs K Abe (Japon), A. Vila (Grenoble), N. Monnier (Grenoble), R. Saura (Bordeaux) et les Prs B. Bady ( $\dagger$ ) (Lyon), B. Echenne (Montpellier), R. Frants (Hollande), G. Lenoir (Lyon), H. Nivet (Tours), C. Piussan (Amiens), J.M. Saudubray (Paris). Nous remercions le Dr D. Recan et le Pr. J.C. Kaplan pour la banque de cellules (Hôpital Cochin, Paris), ainsi que le Généthon (Évry) pour son aide à la cartographie génétique. Nous remercions le Dr N. Baumann ainsi que les Prs Y. Agid et O. Lyon-Caen pour leur aide et leur intérêt pour ce travail. Cette recherche a bénéficié du soutien financier de l'Association Française contre les Myopathies (AFM), du ministère de la Recherche en France (GREG) et en Allemagne.

\section{Summary}

Hypokalemic periodic paralysis and the dyhydropyridine receptor: an unexpected role for a calcium channel!

Periodic paralyses and myotoniae, except myotonic dystrophy, are hereditary diseases of the muscle ion channels. Hyperkalemic periodic paralysis and paramyotonia congenita are sodium channel diseases whereas myotonia congenita is a chloride channelopathy. Hypokalemic periodic paralysis (hypoPP) was the last member of this group of diseases with an unknown gene defect. HypoPP patients present acute and reversible attacks of muscle weakness concomitant to a blood potassium level decrease. HypoPP is a genetic disease transmitted with an autosomal dominant inheritance. The onset of the disease is in the second decade. In the fourth or fif th decade of life appears a vacuolar myopathy responsible for a permanent muscle weakness. By a strategy combining a genome-wide search of the locus and a candidate gene approach, we showed by linkage analysis that the hypoPP locus and a gene coding for a calcium chan- nel, the $\alpha$ l-subunit of the dihydropyridine receptor, co-localize to chromosome lq31-32. We further demonstrated a mutation in segment S4 of domain II: the substitution of an arginine for an histidine in position 528 of the dihydropyridine receptor. A similar mutation was reported by american investigators in position 1239 (segment S4 of domain IV). The two mutations: $\operatorname{Arg}^{528} \rightarrow$ His and $\operatorname{Arg}^{1239} \rightarrow$ His account for a majority of hypoPP cases. The two mutations affect segment S4 which is the voltage-sensor of the calcium channel. Therefore, they might directly explain the acute attacks of paralysis caused by muscle depolarization. However, the direct role of the dihydropyridine receptor in the hypokalemia and the vacuolar myopathy remains to be established. These unanswered questions should benefit from the on-going studies with in vitro expression of mutated dihydropyridine receptors. 